\title{
Production of poly(GA) in C9ORF72 patient motor neurons derived from induced pluripotent stem cells
}

\author{
Sandra Almeida ${ }^{1} \cdot$ Gopinath Krishnan ${ }^{1} \cdot$ Mia Rushe $^{2} \cdot$ Yuanzheng Gu$^{2} \cdot$ Mark W. Kankel $^{2} \cdot$ Fen-Biao Gao ${ }^{1}$ (i)
}

Received: 26 August 2019 / Revised: 8 October 2019 / Accepted: 8 October 2019 / Published online: 17 October 2019

(c) The Author(s) 2019

GGGGCC $\left(\mathrm{G}_{4} \mathrm{C}_{2}\right)$ repeat expansion in the first intron of C9ORF72 is the most common genetic cause of amyotrophic lateral sclerosis (ALS) and frontotemporal dementia (FTD) [3, 9]. A key pathological hallmark of C9ORF72related ALS/FTD is the accumulation of dipeptide repeat (DPR) proteins synthesized from both sense and antisense repeat RNAs in affected neurons [2, 8, 14]. The toxicity of poly(GA), poly(GR) and some other DPR proteins in cell and animal models has been well documented, including in mouse models. However, it is poly(GR) whose distribution in patient brains seems to best correlate with neurodegeneration $[10,11]$.

How DPR proteins are synthesized in human $C 9 O R F 72$ neurons is unknown. One widely cited hypothesis is repeatassociated non-AUG (RAN) translation, which posits a novel mechanism by which ribosomes directly initiate translation on expanded repeats [13]. Over time, the term "RAN translation" has evolved to also refer to the phenomenon, that various expanded repeat sequences such as $\mathrm{G}_{4} \mathrm{C}_{2}$ repeats can be translated in different frames. However, whether this truly represents a novel translation mechanism has not yet been vigorously tested in human neurons.

To investigate how DPR proteins are synthesized in C9ORF72 human neurons, we used CRISPR-Cas9 technology to generate a homozygous deletion in the first intron of $C 9 O R F 72,5^{\prime}$ to the $\mathrm{G}_{4} \mathrm{C}_{2}$ repeats (Figs. 1a, $\mathrm{S} 1$ and $\mathrm{S} 2$ ) to

Electronic supplementary material The online version of this article (https://doi.org/10.1007/s00401-019-02083-z) contains supplementary material, which is available to authorized users.

Sandra Almeida

sandra.almeida@umassmed.edu

Fen-Biao Gao

fen-biao.gao@umassmed.edu

1 Department of Neurology, University of Massachusetts Medical School, Worcester, MA 01605, USA

2 Neuromuscular and Movement Disorders, Biogen, Cambridge, MA 02142, USA assess the effect of this deletion on DPR production. This deletion contains a CUG start codon that was shown to play a role in poly(GA) production in reporter constructs $[4,12]$. The induced pluripotent stem cell (iPSC) line used in this experiment contains $\sim 1000$ copies of the $\mathrm{G}_{4} \mathrm{C}_{2}$ repeats [1]. We selected two iPSC lines containing a deletion in intron 1 and differentiated them and the parental iPSC line into ChAT-positive motor neurons (Figs. 1b and S3) as described [5]. This intronic deletion did not compromise the splicing of the intron containing the expanded repeats, as the levels of mature mRNAs for C9ORF72 variants 1-3 and antisense repeat RNA did not decrease (Fig. 1c-f).

To determine whether this deletion affects DPR production, in addition to the intron deletion and the parental iPSC lines described above, an iPSC line derived from a control subject [1] and an isogenic line in which expanded $\mathrm{G}_{4} \mathrm{C}_{2}$ repeats have been deleted by CRISPR-Cas9 [5] (Fig. 1h) were differentiated three times into motor neurons. After 1.5 months, motor neuron cultures were collected and analyzed for poly(GA) content, in a blinded manner, with a new Meso Scale Discovery (MSD) immunoassay developed at Biogen Inc (see Suppl. Information). We found that poly(GA) production was abolished in neurons containing the intronic deletion (Fig. 1i). The absence of poly(GA) production was not due to the absence of the mRNA translation template, as repeat-containing introns were still spliced (Fig. 1c, e) and the level of the pre-mRNA was not significantly decreased (Fig. 1g). More importantly, both poly(GR) and poly(GP) were still produced (Fig. 1j, k). To confirm these results, we differentiated again the no-repeats, the C9ORF72 parental and the two intronic deletion lines 6 or 3 more times into 3-month-old motor neuron cultures. Once more, blinded poly(GA) measurements confirmed the absence of poly(GA) in intronic deletion lines (Fig. 11), while poly(GR) and poly(GP) production was not decreased (Fig. 1m, n).

Next, we used this model to investigate the contribution of poly(GA) to molecular phenotypes found in C9ORF72 
a

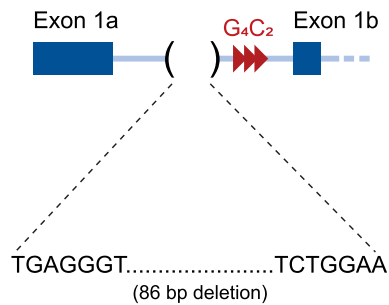

C

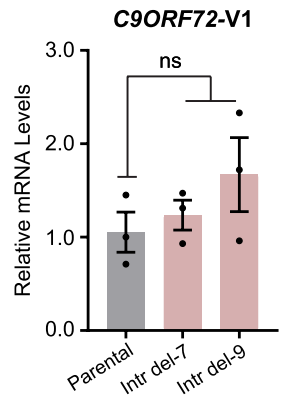

h
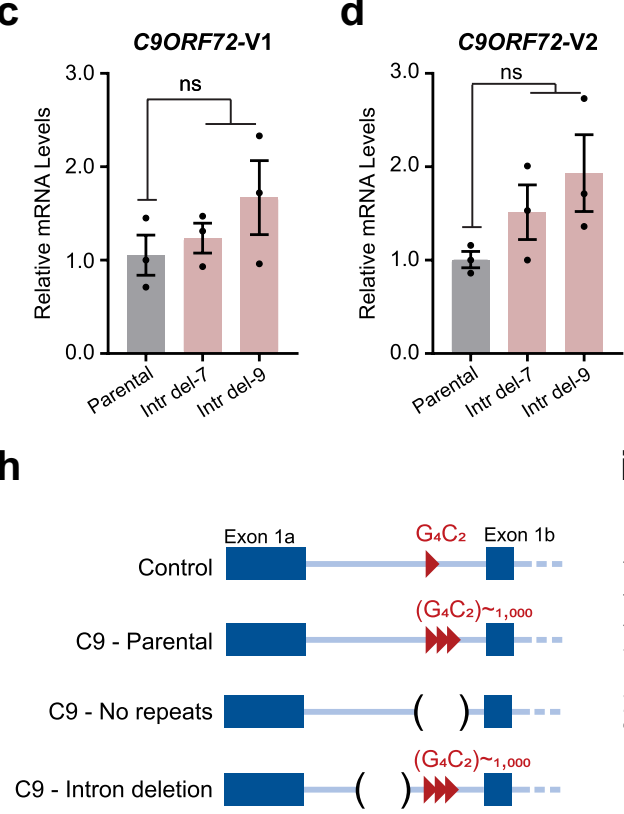

b Location of the intron deletion in C9ORF72
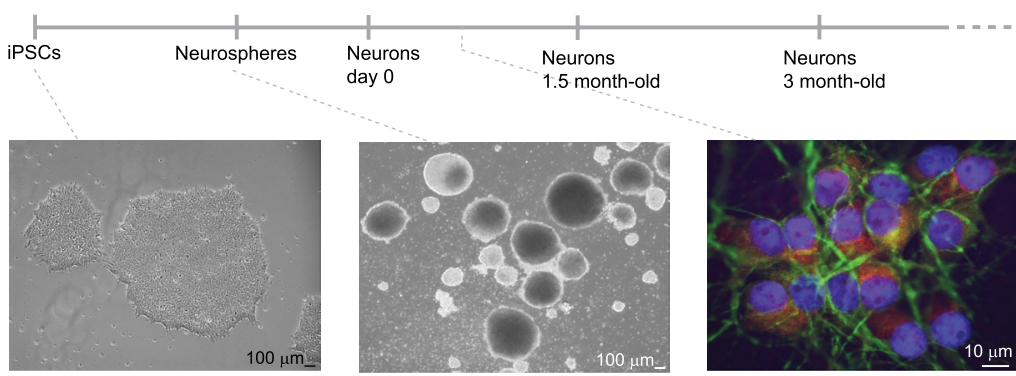

f

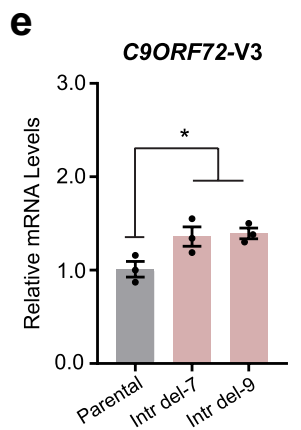

f

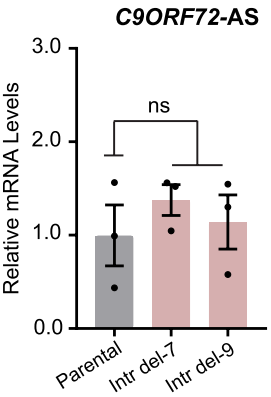

g

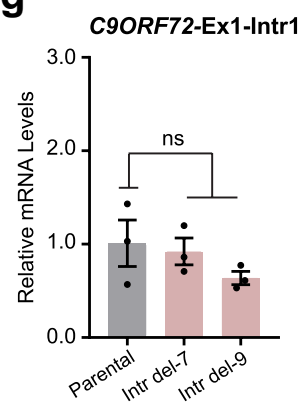

i

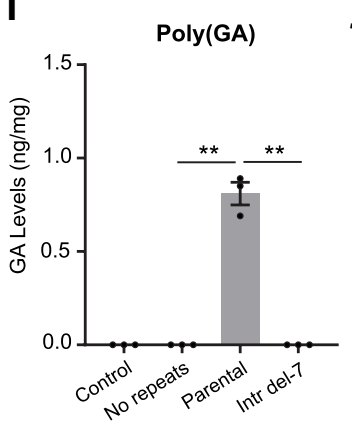

j

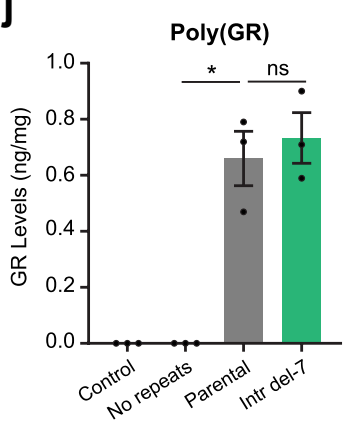

k
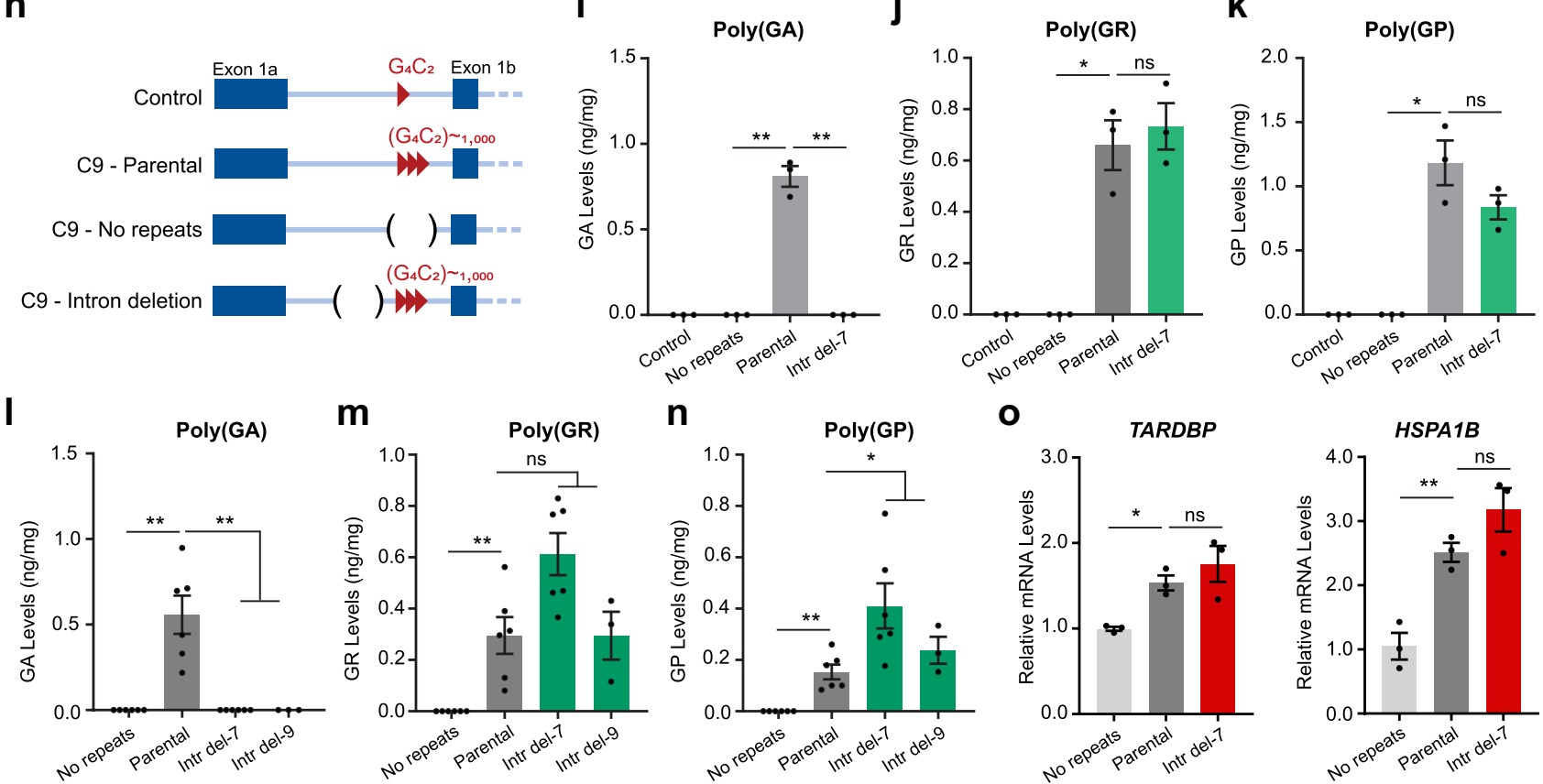

Fig. 1 Poly(GA) production in C9ORF72 iPSC-derived motor neurons. a Schematic of the location of the CRISPR-Cas9-mediated deletion in the first intron of C9ORF72. b Schematic of the motor neurons differentiation protocol. Images show iPSC colonies, neurospheres, and 10-day-old $\mathrm{ChAT}^{+}$(red) and $\mathrm{MAP}^{+}$(green) neurons (DAPI in blue). c-g Neurons from parental and intron deletion lines were analyzed for expression of C9ORF72-V1, C9ORF72-V2, C9ORF72V3, C9ORF72-antisense mRNAs, and C9ORF72 pre-mRNA, $n=3$ independent differentiations. h Schematic of the C9ORF72 locus in four types of iPSC-derived neurons. i-n Poly(GA), poly(GR), and poly(GP) levels in 1.5-month-old ( $n=3$ independent differentiations) and 3-month-old ( $n=3$ or 6 independent differentiations) control, parental, and deletion motor neurons measured by MSD immunoassays. o Relative expression levels of TARDBP and HSPA1B in parental and intron deletion lines, $n=3$ independent differentiations. In all panels, each data point represents one independent differentiation. Values are mean \pm SEM. $* p<0.05, * * p<0.01$ (c-g and $\mathbf{o}$, one-way ANOVA; i-n, Welch's t-test) 
iPSC-derived neurons or patient brain tissues. Upregulation of the expression of heat shock proteins and TAR DNA binding protein 43 (TDP-43) have been reported in ALS/ FTD patient brain tissues [6, 7]. Here, we show that both $H S P A 1 B$ and TARDBP mRNA are upregulated in 4-monthold motor neuron cultures (Fig. 1o). However, in the absence of poly(GA), these molecular phenotypes were not rescued, suggesting that, at least for these phenotypes in this experimental system, poly(GA) was not a key toxic DPR protein.

Our results show that in $C 9 O R F 72$ patient neurons, $\sim 1000$ copies of $\mathrm{G}_{4} \mathrm{C}_{2}$ repeats alone are not sufficient to direct ribosome entry to initiate translation of poly(GA) frame. What remains to be determined is whether other cis elements within the first intron initiate the synthesis of poly(GR) and other DPR proteins or whether their production results from bona fide "RAN translation" in C9ORF72 patient neurons. These mechanistic investigations will help in the design of therapeutic approaches that aim to decrease DPR protein production.

Acknowledgements This work was supported by grants from the NIH (R37NS057553, R01NS101986, and R01NS093097 to FBG and R21NS112766 to SA), the MDA Foundation and the Target ALS Foundation (F-BG), as well as the Frick Foundation for ALS Research, Alzheimer's Association (2016-NIRG-396129) and ALS Association (SA).

Open Access This article is distributed under the terms of the Creative Commons Attribution 4.0 International License (http://creativeco mmons.org/licenses/by/4.0/), which permits unrestricted use, distribution, and reproduction in any medium, provided you give appropriate credit to the original author(s) and the source, provide a link to the Creative Commons license, and indicate if changes were made.

\section{References}

1. Almeida S, Gascon E, Tran H, Chou HJ, Gendron TF, Degroot S et al (2013) Modeling key pathological features of frontotemporal dementia with C9ORF72 repeat expansion in iPSC-derived human neurons. Acta Neuropathol 126:385-399

2. Ash PE, Bieniek KF, Gendron TF, Caulfield T, Lin WL, Dejesus-Hernandez $\mathrm{M}$ et al (2013) Unconventional translation of C9ORF72 GGGGCC expansion generates insoluble polypeptides specific to c9FTD/ALS. Neuron 77:639-646
3. DeJesus-Hernandez M, Mackenzie IR, Boeve BF, Boxer AL, Baker M, Rutherford NJ et al (2011) Expanded GGGGCC hexanucleotide repeat in noncoding region of C9ORF72 causes chromosome 9p-linked FTD and ALS. Neuron 72:245-256

4. Green KM, Glineburg MR, Kearse MG, Flores BN, Linsalata AE, Fedak SJ et al (2017) RAN translation at C9orf72-associated repeat expansions is selectively enhanced by the integrated stress response. Nat Commun 8:2005

5. Lopez-Gonzalez R, Yang D, Pribadi M, Kim TS, Krishnan G, Choi SY et al (2019) Partial inhibition of the overactivated Ku80dependent DNA repair pathway rescues neurodegeneration in C9ORF72-ALS/FTD. Proc Natl Acad Sci USA 116:9628-9633

6. Mishra M, Paunesku T, Woloschak GE, Siddique T, Zhu LJ, Lin $S$ et al (2007) Gene expression analysis of frontotemporal lobar degeneration of the motor neuron disease type with ubiquitinated inclusions. Acta Neuropathol 114:81-94

7. Mordes DA, Prudencio M, Goodman LD, Klim JR, Moccia R, Limone $F$ et al (2018) Dipeptide repeat proteins activate a heat shock response found in C9ORF72-ALS/FTLD patients. Acta Neuropathol Commun 6:55

8. Mori K, Weng SM, Arzberger T, May S, Rentzsch K, Kremmer E et al (2013) The C9orf72 GGGGCC repeat is translated into aggregating dipeptide-repeat proteins in FTLD/ALS. Science 339:1335-1338

9. Renton AE, Majounie E, Waite A, Simon-Sanchez J, Rollinson S, Gibbs JR et al (2011) A hexanucleotide repeat expansion in C9ORF72 is the cause of chromosome 9p21-linked ALS-FTD. Neuron 72:257-268

10. Saberi S, Stauffer JE, Jiang J, Garcia SD, Taylor AE, Schulte D et al (2018) Sense-encoded poly-GR dipeptide repeat proteins correlate to neurodegeneration and uniquely co-localize with TDP43 in dendrites of repeat-expanded C9orf72 amyotrophic lateral sclerosis. Acta Neuropathol 135:459-474

11. Sakae N, Bieniek KF, Zhang YJ, Ross K, Gendron TF, Murray ME et al (2018) Poly-GR dipeptide repeat polymers correlate with neurodegeneration and clinicopathological subtypes in C9ORF72related brain disease. Acta Neuropathol Commun 6:63

12. Tabet R, Schaeffer L, Freyermuth F, Jambeau M, Workman M, Lee CZ et al (2018) CUG initiation and frameshifting enable production of dipeptide repeat proteins from ALS/FTD C9ORF72 transcripts. Nat Commun 9:152

13. Zu T, Gibbens B, Doty NS, Gomes-Pereira M, Huguet A, Stone MD et al (2011) Non-ATG-initiated translation directed by microsatellite expansions. Proc Natl Acad Sci USA 108:260-265

14. Zu T, Liu Y, Banez-Coronel M, Reid T, Pletnikova O, Lewis J et al (2013) RAN proteins and RNA foci from antisense transcripts in C9ORF72 ALS and frontotemporal dementia. Proc Natl Acad Sci USA 110:E4968-4977

Publisher's Note Springer Nature remains neutral with regard to jurisdictional claims in published maps and institutional affiliations. 